Article

\title{
Design and Applications of In-Cavity Pulse Shaping by Spectral Sculpturing in Mode-Locked Fibre Lasers
}

\author{
Sonia Boscolo ${ }^{1, *}$, Junsong Peng ${ }^{1}$ and Christophe Finot ${ }^{2}$ \\ ${ }^{1}$ Aston Institute of Photonic Technologies, School of Engineering and Applied Science, \\ Aston University, Birmingham B4 7ET, UK; E-Mail: j.peng@ aston.ac.uk \\ ${ }^{2}$ Laboratoire Interdisciplinaire Carnot de Bourgogne, UMR 6303 CNRS-Université de \\ Bourgogne/Franche-Comté, 9 avenue Alain Savary, BP 47870, 21078 Dijon Cedex, France; \\ E-Mail: christophe.finot@u-bourgogne.fr \\ * Author to whom correspondence should be addressed; s.a.boscolo@aston.ac.uk; \\ Tel.: +44-121-204-3495; Fax: +44-121-204-3682.
}

Academic Editor: Totaro Imasaka

Received: 20 October 2015 / Accepted: 13 November 2015 / Published: 25 November 2015

\begin{abstract}
We review our recent progress on the realisation of pulse shaping in passively-mode-locked fibre lasers by inclusion of an amplitude and/or phase spectral filter into the laser cavity. We numerically show that depending on the amplitude transfer function of the in-cavity filter, various regimes of advanced waveform generation can be achieved, including ones featuring parabolic-, flat-top- and triangular-profiled pulses. An application of this approach using a flat-top spectral filter is shown to achieve the direct generation of high-quality sinc-shaped optical Nyquist pulses with a widely tunable bandwidth from the laser oscillator. We also present the operation of an ultrafast fibre laser in which conventional soliton, dispersion-managed soliton (stretched-pulse) and dissipative soliton mode-locking regimes can be selectively and reliably targeted by adaptively changing the dispersion profile and bandwidth programmed on an in-cavity programmable filter. The results demonstrate the strong potential of an in-cavity spectral pulse shaper for achieving a high degree of control over the dynamics and output of mode-locked fibre lasers.
\end{abstract}

Keywords: mode-locked fibre lasers; nonlinear pulse shaping; frequency filtering; nonlinear fibre optics 


\section{Introduction}

Passively-mode-locked fibre lasers have been the subject of considerable research interest for several decades owing to their potential for realising reliable and cost-effective compact ultrafast light sources [1-3]. While being very practical devices, mode-locked lasers are still very interesting objects for fundamental research in nonlinear science. From a fundamental standpoint, mode-locked fibre lasers provide convenient and reproducible experimental settings for the study of a variety of nonlinear dynamical processes thanks to the relative simplicity and precision of fibre-optic experiments. Nonlinear effects are usually quite large in mode-locked fibre lasers, but the interplay among the effects of gain/loss, dispersion and nonlinearity can also be used to shape the pulses, manipulate and control the light dynamics and, hence, lead to different regimes of mode locking. Five distinct nonlinear regimes of pulse generation have been demonstrated in passively-mode-locked fibre lasers, including soliton [4] and stretched-pulse [5,6] regimes, self-similar pulse (similariton) propagation in passive [7] and active [8-11] fibres and all-normal or strong net-normal dispersion regimes [12-16]. These mode-locking dynamics have been observed at the emission wavelengths of both ytterbium and erbium, as well as in Raman fibre oscillators and, more recently, also in thulium-doped fibre lasers [17], so they are not specific to the particular gain medium. A conventional soliton formed by the balance of self-phase modulation (SPM) and negative group-velocity dispersion (GVD) is chirp free and propagates unchanged, but its pulse energy is limited to a low level by the soliton area theorem. In a laser with segments of large and nearly equal magnitudes of GVD, but with opposite signs (referred to as a dispersion map), a pulse will stretch and compress, and the nonlinear phase is exactly balanced by the net effect of dispersion. This stretched-pulse or dispersion-managed (DM) soliton operation exists for net anomalous or small normal GVD and allows ultrashort pulses with up to nanojoule energies. Recent work [18] has shown that much higher pulse energies can be achieved in fibre lasers that operate at large normal dispersion. In the normal-dispersion regime, to compensate the nonlinear phase and avoid wave-breaking phenomena, dissipation is required and plays a key role in the pulse shaping. The similariton regime existing in DM cavities with large normal GVD features parabolic pulses that evolve self-similarly in a long segment of passive fibre [7]. These pulses linearise the nonlinear phase in the normal-dispersion fibre, which is compensated by an anomalous GVD segment. Oscillators supporting amplifier similaritons [8-11] rely on a local nonlinear attraction to stabilise the pulse in the cavity, while spectral filtering plays an important supporting role by undoing the large spectral broadening after the gain segment. It is worth mentioning that the soliton-similariton laser reported in [8,9] is the only example of a mode-locked laser so far that has two types of nonlinear waves propagating within the cavity. In cavities with only normal-dispersion components or strong net-normal dispersion, the resulting dissipative solitons (DSs) balance nonlinear phase accumulations by spectral or gain filtering of a highly-chirped pulse in the laser cavity. To date, the best energy performance from standard single-mode fibre (SMF) lasers has been achieved with this mode-locking mechanism.

Techniques for generating, controlling and manipulating ultrashort optical pulses and specialised waveforms [19] have become increasingly important in many scientific areas, including, amongst others, ultra-high-speed optical communications, optical signal processing and biophotonics. Versatile ultrafast laser sources, which can selectively emit different types of nonlinear waves, are highly desirable in this 
context. Different regimes of pulse generation at net anomalous or normal dispersion can be realised in a DM fibre laser via appropriate in-cavity dispersion management [20-22]. Commonly-employed methods to achieve flexible control of in-cavity dispersion include grating pairs, prisms or simply physically changing the length of the fibre in the cavity [20-23]. Furthermore, the possibility to achieve both parabolic self-similar and triangular pulse shaping in a mode-locked fibre laser via adjustment of the net normal dispersion and integrated gain of the cavity was reported in [24]. In [25], careful control of the gain/loss parameters of a net-normal dispersion laser cavity provided the means of achieving switching among Gaussian pulse, DS and similariton pulse solutions in the cavity. All of these techniques, however, require manual tuning of some physical parameters of the cavity.

Spectral pulse shaping [26] is a technique that employs spectral manipulation of the intensity and phase components of a pulse in order to create the desired field distribution. It has been highly successful for applications in a number of fields [27,28]. However, since the pulse shaper is normally placed outside a laser cavity, it requires careful alignment between the wavelength of the shaping filter and the emission wavelength of the laser, and its control of the laser field is limited; the bandwidth of the filtered spectrum is determined by the bandwidth of the input spectrum, and manipulation of the in-cavity dynamics of the laser is not possible. In addition, the pulse shaper can only subtract power from the frequency components of the signal while manipulating its intensity, thereby potentially making the whole process extremely power inefficient. Fully-programmable amplitude and phase filters [29] are already commercially available and extensively used in telecommunications applications [30]. The use of such filters, when placed inside a laser cavity, has the potential to allow the operation of lasers that exhibit pulse characteristics that can be controlled purely through software control. The inclusion of a pulse shaper into the cavity of a passively-mode-locked fibre laser was recently demonstrated [31], allowing for the tunability of the laser wavelength and operation at high repetition rates. In [32,33], the phase-filtering ability of an in-cavity pulse shaper was shown to enable precise control of the cavity dispersion of the laser, as well as to change the output pulse train from bright to dark pulses. In this paper, we review our recent results and advances in the area, by numerically describing a novel approach to achieving different regimes of pulse generation in a passively-mode-locked fibre laser, which relies on nonlinear in-cavity pulse dynamics and pulse shaping by an in-cavity amplitude and phase spectral filter [34]. By changing the amplitude profile applied on the filter, we are able to create various output temporal waveforms of fundamental and practical interest, ranging from bright and dark parabolic profiles to a flat-top profile and triangular and saw-tooth (asymmetric triangular) profiles [35]. As an application of this general approach, we show that the use of an in-cavity flat-top spectral filter makes it possible to directly generate sinc-shaped Nyquist pulses of high quality and of a widely-tunable bandwidth from the laser [36]. We also report on a recently-developed versatile erbium-doped fibre (EDF) laser, in which conventional soliton, DM soliton and DS mode-locking regimes can be selectively and reliably targeted by programming different GVD profiles and bandwidths on an in-cavity programmable filter [37]. 


\section{In-Cavity Pulse Shaping}

\subsection{Design Rationale and Numerical Model}

The design of in-cavity pulse shaping by spectral filtering exploits the understanding gained by our recent work [34]. The cavity configuration considered is a simple ring cavity, as shown in Figure 1. A short (a few meters) segment of rare-earth doped fibre with normal GVD acts as the gain and nonlinear element of the cavity. The gain fibre is followed by a saturable absorber (SA) element initiating and stabilising the mode-locked operation and an amplitude and phase spectral filter, which realises the pulse shaping. Note that for the Nyquist pulse shaping described thereafter, the positioning of the SA and filter in the cavity is reversed, as we found that coupling the filter to receive output light from the fibre amplifier enabled a wider tunability range for the filter bandwidth. The output of the laser is monitored behind a coupler at the output of the filter. Pulse propagation in the laser cavity can be described by a theoretical model that includes the dominant physical effects of the system on the evolution of a pulse over one round trip inside the cavity [38]. These effects include GVD, SPM, gain saturation and bandwidth-limited gain for the active fibre, as well as the discrete effects of the SA and spectral filter. The evolution in the fibre section is described by a standard modified nonlinear Schrödinger equation [38]:

$$
i \psi_{z}-\frac{1}{2} \beta_{2} \psi_{t t}+\gamma|\psi|^{2} \psi=\frac{i}{2} g\left(\psi+\frac{1}{\Omega^{2}} \psi_{t t}\right)
$$

where $\psi=\psi(z, t)$ is the slowly-varying amplitude of the pulse envelope, $z$ and $t$ are the propagation and time delay parameters, $\beta_{2}$ is the GVD parameter and $\gamma$ is the coefficient of the cubic nonlinearity of the fibre. The gain is saturated according to $g=g(z)=g_{0} /\left(1+W / W_{0}\right)$, where $g_{0}$ is the small-signal gain, $W(z)=\int \mathrm{d} t|\psi|^{2}$ is the pulse energy and $W_{0}$ is the gain saturation energy determined by the pump power. The action of the fast SA is approximated by a simplified, but generic, nonlinear transfer function describing its transmittance [38]: $T=1-q_{0} /\left[1+P(t) / P_{0}\right]$, where $q_{0}$ is the unsaturated loss due to the absorber, $P(z, t)=|\psi(z, t)|^{2}$ is the instantaneous pulse power and $P_{0}$ is the saturation power. The filter is modelled by the spectral response $H(f)=R(f) \exp \left[i \beta_{2 \text {,acc }}(2 \pi f)^{2} / 2\right]$, where $R(f)$ is the Fourier transform of a certain objective waveform function $r(t)$, and the spectral phase adds a specific amount of GVD to the cavity. By varying the curvature $\beta_{2 \text {,acc }}\left(\right.$ in $\mathrm{ps}^{2}$ ) of the parabolic phase function, we can control the net dispersion of the cavity. The numerical model is solved with a standard symmetric split-step propagation algorithm, and the initial field is a picosecond Gaussian temporal profile. The following parameters, typical of ytterbium-doped fibres [7], are used in the simulations: $\beta_{2}=25 \mathrm{fs}^{2} / \mathrm{mm}$, $\gamma=0.005(\mathrm{~W} \mathrm{~m})^{-1}$, and $\Omega$ corresponds to a 40-nm bandwidth at FWHM. Furthermore, $g_{0}$ corresponds to $30 \mathrm{~dB} / \mathrm{m}$ in power, $W_{0}=150 \mathrm{pJ}, P_{0}=150 \mathrm{~W}$, and $70 \%$ of laser power is tapped out of the cavity.

The main pulse-shaping mechanism of our laser design relies on enhanced spectral filtering of a nonlinearly spectrally-broadened pulse in the cavity [34]. A significant nonlinear phase shift is impressed on the pulse in the fibre section. The spectral filter compensates both the broad pulse duration and bandwidth after the fibre segment. The principle of filtering a strongly-phase-modulated pulse both in frequency and time has already been exploited to achieve new mode-locking regimes in fibre lasers [8,10]. In [34], we extended this approach to more complex features. Because the nonlinearly-broadened spectrum of the pulse at the output of the fibre is wider than the filter's spectral response and fairly flat within the filter bandwidth (see Figure 2), it can be sliced without caring for the 
details of its structure [39]. This is the enabling mechanism for the formation of pulses of the desired temporal shape at the output of the filter, impressed on the pulse by the filter's impulse response. Note that unlike the cavity design presented in [34], here, we do not employ an additional passive nonlinear propagation stage in the cavity. This notwithstanding, the spectral broadening in the gain fibre segment is still sufficient to operate a convenient reshaping. It is also worth noting that steady-state pulse shaping in an oscillator requires the generated pulse to fulfil the constraints imposed by the cavity, and the formation of a self-consistent solution in the cavity is in general a non-trivial problem. Despite substantial research in this field, qualitatively new phenomena are still being unveiled.

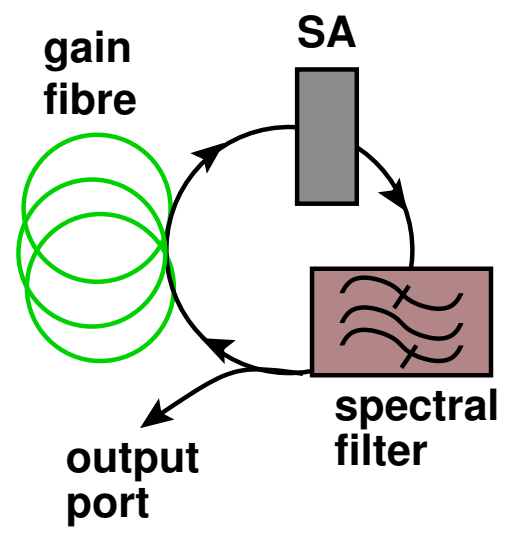

Figure 1. Schematic of the laser. SA: saturable absorber.
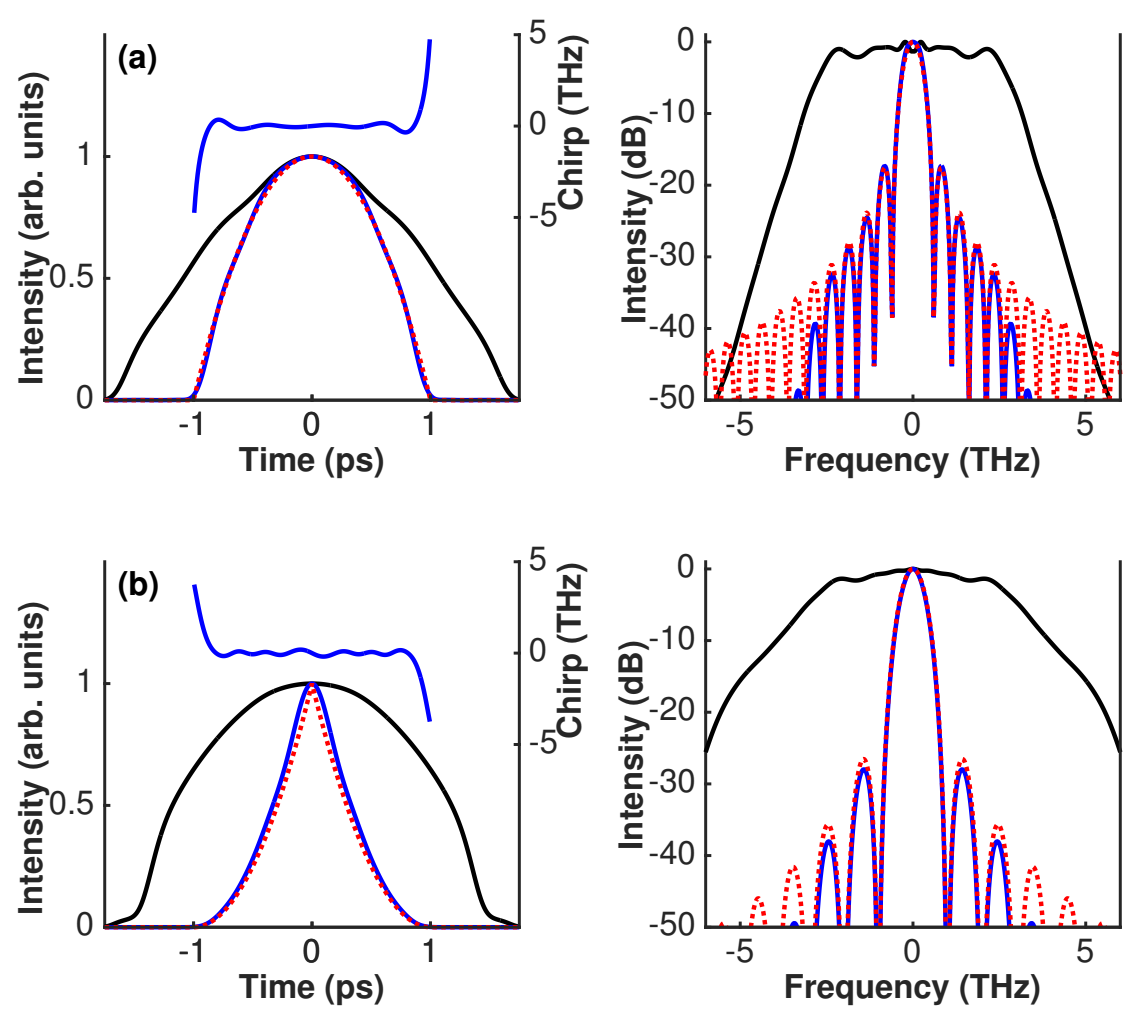

Figure 2. Cont. 

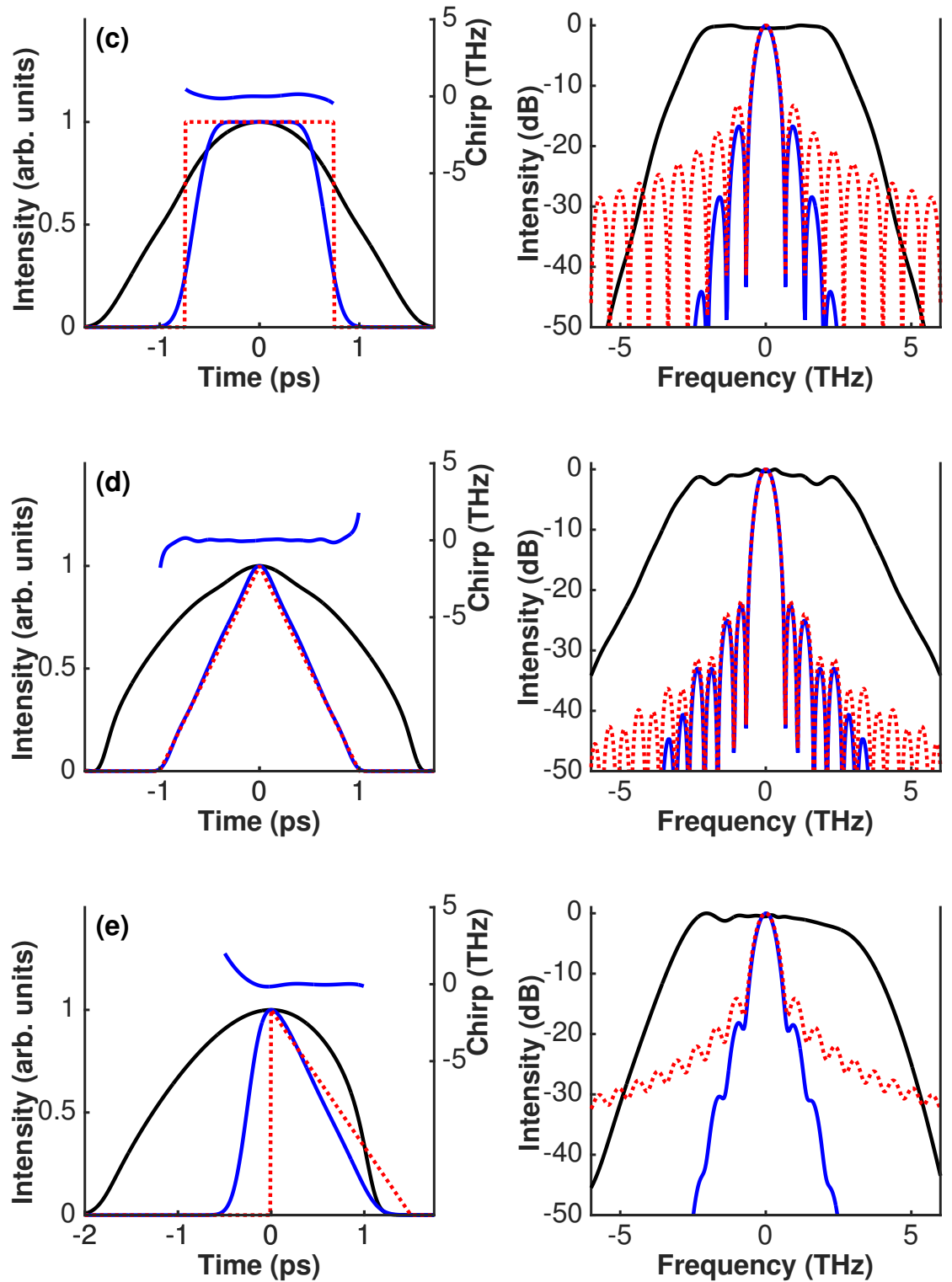

Figure 2. Temporal intensity and chirp profiles (left) and spectral intensity profiles (right) of the output pulse from the laser (blue) for a (a) bright parabolic, (b) dark parabolic, (c) flat-top, (d) triangular and (e) saw-tooth impulse response of the spectral pulse shaper. Also shown are the target profiles (red dotted) and the profiles at the exit of the gain fibre (black).

\subsection{Tailored Pulse Waveform Generation}

As an illustration of the general pulse-shaping principle described above, we consider here the generation of five different amplitude functions [34], which represent some examples of interesting optical waveforms for applications in optical signal processing and manipulation [35]. We use a bright parabolic pulse-shaping filter with $r(t)=\sqrt{1-(t / \tau)^{2}} \theta(\tau-|t|), \tau=1 \mathrm{ps}$; a dark parabolic pulse shaper, such that $r(t)=(1-|t / \tau|) \theta(\tau-|t|)$ with $\tau=1 \mathrm{ps}$; a flat-top pulse shaper where $r(t)=\theta(\tau-|t|)$ with $\tau=0.75 \mathrm{ps}$; a triangular pulse shaper where $r(t)=\sqrt{1-|t / \tau|} \theta(\tau-|t|)$ with $\tau=1 \mathrm{ps}$; and a saw-tooth pulse shaper, where we define $r(t)=\sqrt{1-t / \tau}$ in $t \in[0, \tau], r(t)=0$, otherwise, with $\tau=1.5 \mathrm{ps}$. Here, 
$\theta(x)$ is the Heaviside function. The spectral filter can be realised in a variety of ways, such as a fibre Bragg grating or even a programmable liquid-crystal-on-silicon (LCoS) optical processor, which offers the additional advantage of being easily reconfigurable [29].

The pulse solutions obtained for the different amplitude profiles applied on the filter and net GVD of $0.008 \mathrm{ps}^{2}$ are shown in Figure 2. It is seen that the desired pulse shapes are obtained at the output of the laser. In the case of flat-top and saw-tooth waveform functions, because of the sharp edges of these pulse shapes, an additional Gaussian bandpass filter with a bandwidth at FWHM of $1.7 \mathrm{THz}$ is included in the pulse shaper to make the top of the resulting pulse ripple-free and to smooth the formed pulse shape, respectively. The generated pulses are nearly transform-limited. However, as opposed to the chirp (instantaneous frequency shift) profile targeted by the filter, the chirp profile of the generated pulses may exhibit a small ripple. This indicates that generating pulses with a well-defined phase profile by use of the method described here is not as straightforward. The pulse evolution, illustrated in Figure $3 \mathrm{a}$ by plots of the FWHM pulse duration and spectral bandwidth as functions of position in the cavity, highlights the basis of the pulse-shaping mechanism being used. The evolution shown for the triangular pulse-shaping regime is representative of those observed for the other shaping regimes, too, in which the typical pulse parameters evolve in a closely-similar fashion along the cavity, though small quantitative differences may exist. It is seen that the nonlinear evolution in the gain fibre is monotonic with the growth of temporal and spectral widths, while the pulse acquires a positive (normal) chirp. The filter compensates for temporal and spectral broadening and cancels the temporal phase accumulation in the fibre. Although the SA is not significant in shaping the pulse, it improves the stability of the laser by attenuating low-amplitude wings that can grow over many cavity round trips. The peak nonlinear phase shift $\phi^{\mathrm{NL}}=\int \mathrm{d} z \gamma P_{p}(z)$ ( $P_{p}$ is the pulse peak power), which the pulse accumulates in the gain fibre (also known as the $B$-integral), is approximately $12 \mathrm{rad}$ in the example of Figure $3 \mathrm{a}$. This is accommodated by a spectral breathing factor (defined as the ratio of the spectral width at FWHM at the input and the output of the filter) of approximately nine. This confirms that the intended pulse shaping in our laser design is achieved through enhanced spectral filtering of a nonlinearly-broadened pulse in the cavity [34].

To gain a broader understanding of the laser dynamics, we investigated the effect of net dispersion on the laser response. The results [34] revealed that the potential of tuning the output pulse temporal duration through in-cavity linear chirping is minimal. This stems from the filtering process being used, i.e., slicing of a fairly broader spectrum, and indicates that one should act directly on the impulse response function of the filter to control the output pulse width. This result is not surprising, in fact, as a triangular, parabolic or flat-top temporal profile is rather sensitive to group delay. Note that, as we shall see in Section 3, the net dispersion is controlled by adjusting the transfer function of the pulse shaper [29]; therefore, no physical change in the cavity is required in order to change the output from one pulse shape to another, provided that a programmable pulse shaper is used. 

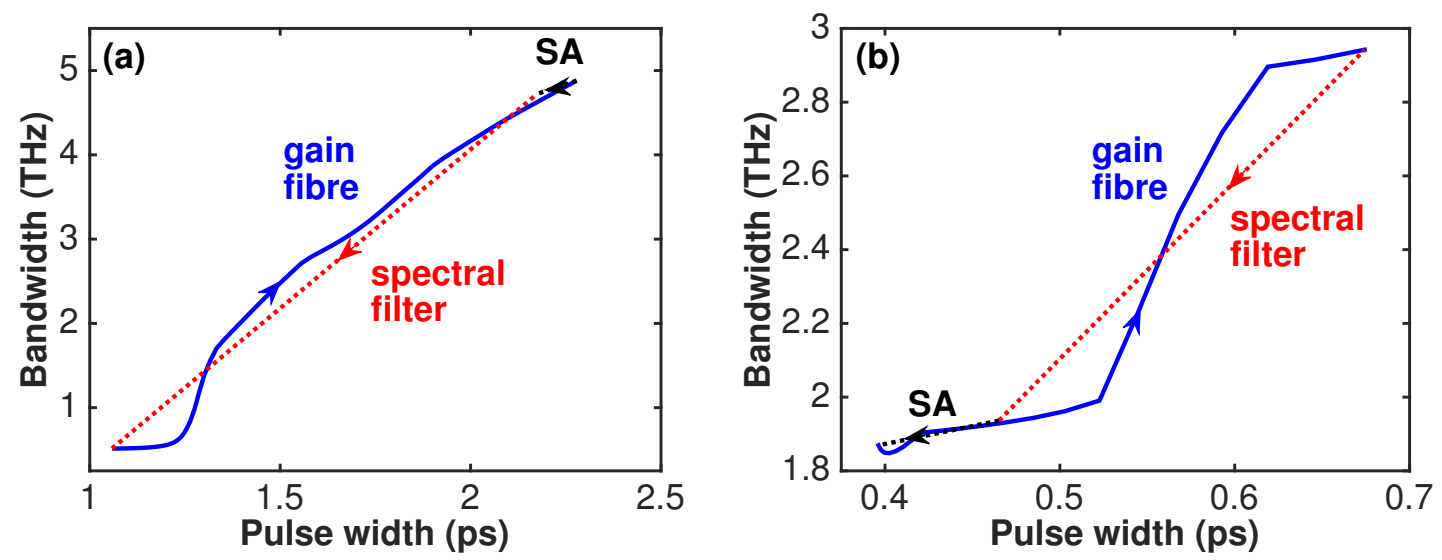

Figure 3. Phase diagrams representing the combined evolution of the FWHM temporal and spectral widths of the pulse over one round-trip in the cavity for: (a) a spectral pulse shaper with a triangular impulse response and a net cavity dispersion of $0.008 \mathrm{ps}^{2}$; (b) a flat-top spectral pulse shaper with $B=2 \mathrm{THz}\left(\tau_{p}=0.5 \mathrm{ps}\right)$ and in the absence of in-cavity dispersion control.

\subsection{Bandwidth Programmable Optical Nyquist Pulse Generation}

In this section, we describe the application of our method for in-cavity pulse shaping to the generation of optical Nyquist pulses [36]. The rectangular spectrum of sinc-shaped Nyquist pulses enables bandwidth-efficient encoding of data, which is of key interest for high-capacity optical communication systems (see, e.g., [40] and the references therein). Besides optical communications, sinc pulses can bring benefits to many other fields, such as all-optical sampling [41], microwave photonics [42] and light storage [43]. Various techniques to optically generate Nyquist pulses have been demonstrated, including spectral reshaping of a mode-locked laser [44] or fibre optical parametric amplification [45]. Additionally, sequences of very high-quality sinc-shaped pulses were obtained by the direct synthesis of a phase-locked frequency comb [46]. Furthermore, a Nyquist laser that can directly emit an optical Nyquist pulse train was recently demonstrated [47]. In the laser design reported in [47], the generation of Nyquist pulses is made possible by a combination of parabolic time-domain shaping at the pulse peak with an optical intensity modulator and spectral-domain shaping on the pulse wings with a spectral filter. In our passively-mode-locked laser design, the incorporation of a simple flat-top spectral filter in the cavity makes it possible to generate sinc-shaped Nyquist pulses of high quality. Furthermore, the use of a filter's profile with a corrective convex top can compensate for the concavity of the pulse spectrum obtained after nonlinear expansion in the fibre, thereby relaxing the need for large nonlinear phase accumulation in the fibre. The simplicity of our fibre laser design is appealing for implementation in experiments. It is typical in optical communications that the availability of a certain technical solution refocuses research on the feasibility of simpler or more cost-efficient versions. 
For the demonstration of Nyquist pulse generation, the amplitude profile $R(f)$ of the filter's spectral response is chosen to have sharply-decaying edges outside its bandwidth and a Gaussian top,

$$
R(f)=\left\{\begin{array}{cc}
\frac{\tau_{p}}{2} \exp \left(\alpha f^{2}\right), & |f|<\frac{1}{\tau_{p}} \\
\frac{\tau_{p}}{4}, & |f|=\frac{1}{\tau_{p}} \\
0 & \text { otherwise }
\end{array}\right.
$$

where $B=2 / \tau_{p}$ is the spectral bandwidth (defined as the width of both edges of the spectrum tail), $\tau_{p}$ is the time interval between zero crossings of the corresponding sinc-function-like impulse response and $\alpha$ is an adjustable parameter defining the width and concavity of the spectrum top. Note that approximating the Gaussian function by the first two terms of its Taylor series, i.e., by a quadratic function, in the filter's response would yield similar results to those presented hereafter. For the special value $\alpha=0, R(f)$ is the rectangular spectral profile associated with the Nyquist sinc-function impulse response [44]:

$$
r(t)=\frac{\sin \left(2 \pi t / \tau_{p}\right)}{2 \pi t / \tau_{p}}
$$

while $\alpha>0(\alpha<0)$ yields a convex or inverted (concave or conventional) Gaussian top. A variety of filters with very steep edges and adjustable bandwidth are readily available. The corrective Gaussian-top profile can be provided by a programmable LCoS optical processor, the shape and bandwidth of which can be software configured [29]. Note that optimisation of the filter's profile is relatively easy as it requires adjustment of only one parameter $(\alpha)$. Note also that the sinc function in Equation (3) defines a time unlimited pulse and, as a consequence of causality, is therefore only a theoretical construct. Instead, a pulse train as generated in the laser would be used in an experimental demonstration of Nyquist pulse shaping. However, given that the pulse repetition period would be significantly larger than the characteristic pulse width $\tau_{p}$, only a negligibly small side-band field would be created by the sinc function between adjacent pulses.

The solutions obtained for a flat-top spectral profile $(\alpha=0)$ applied on the filter with different related zero-crossing pulse durations are shown in Figure 4, and compared to the solutions obtained with a convex Gaussian-top profile. The shape correction factor $\alpha$ was optimised for each pulse duration, and no in-cavity dispersion control was used in these simulations. It is seen that the use of the basic rectangular shape for the filter's spectral response already allows us to obtain sinc-shaped pulses at the output of the laser that coincide well with the theoretical ones described by Equation (3) and feature a spectrum that is fairly close to the ideal rectangular case. When a convex spectral profile is applied to the filter, the correction of the non-perfectly-flattened pulse spectrum after the gain fibre by the filter brings about sinc pulses of enhanced quality and with an almost ideal rectangular spectrum at the laser output for all of the pulse durations being considered. The example of pulse evolution shown in Figure $3 \mathrm{~b}$ when the basic rectangular filtering method is employed demonstrates that the pulses are shaped by the same mechanism as that governing the pulse generation regimes described in the previous section (Figure $3 a$ ). The initial slight decrease of the spectral bandwidth of the pulse in the gain fibre originates from the reshaping of the spectrum from a rectangular-like profile at the entrance of the fibre to a parabolic-like shape near the peak with a transition to a steep decay in the first stage of evolution in the fibre. After this stage, the spectrum broadens significantly and eventually develops the steep and structured edges characteristic of SPM. We also note that while the FWHM pulse duration decreases owing to spectral 
filtering, the root-mean-square (rms) duration is increased by the filter owing to the specific ringing feature (or ripples) on the pulse wings accompanied by the generated Nyquist pulse. The SA decreases the pulse duration and attenuates the pulse ripples. The evolution in the laser exhibits a spectral breathing factor of approximately two, which accommodates a $B$-integral of approximately 3 rad in the example of Figure $3 \mathrm{~b}$. The reduced spectral breathing and nonlinear phase shift accumulation compared to the results of the previous section stem from the shorter length of fibre used in the laser cavity for the Nyquist mode of operation.
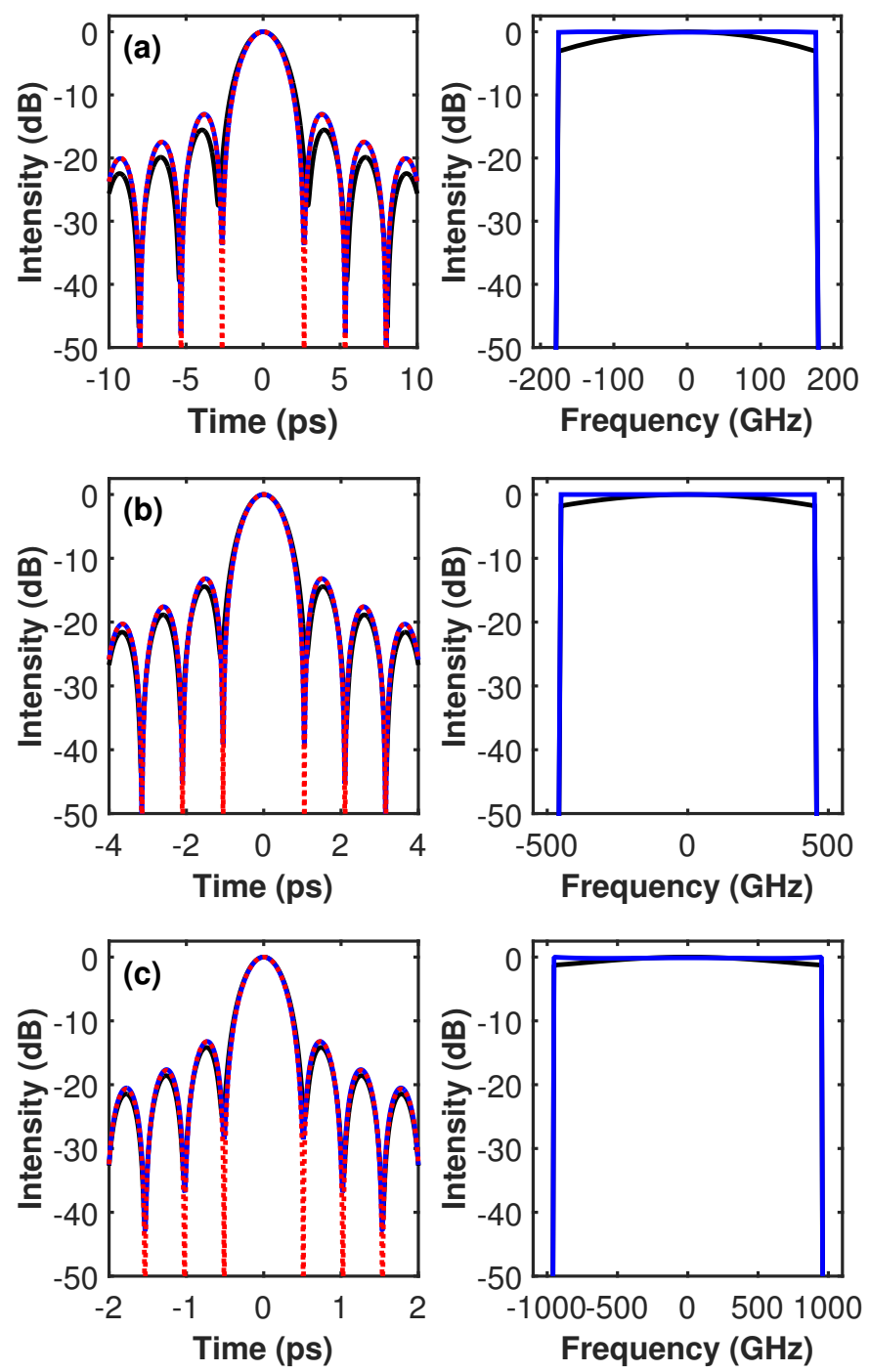

Figure 4. Temporal (left) and spectral (right) intensity profiles of the output pulse from the laser for different filter bandwidths: (a) $B=400 \mathrm{GHz}\left(\tau_{p}=2.5 \mathrm{ps}\right.$ ); (b) $B=1 \mathrm{THz}$ ( $\left.\tau_{p}=1 \mathrm{ps}\right)$; and (c) $B=2 \mathrm{THz}\left(\tau_{p}=0.5 \mathrm{ps}\right)$. The profiles obtained with a flat-top $(\alpha=0)$ spectral pulse shaper (black) are compared to those obtained with a convex Gaussian-top spectral shaper (blue). The correction factor is $\alpha=6.1,1.1,0.18$ in (a-c), respectively. Also shown are the calculated waveforms according to (3) (red dotted). A laser without dispersion control in the cavity is modelled. Adapted from [36]. Copyright 2015, IEEE. 
In order to illustrate the flexibility of the proposed scheme in terms of output spectral bandwidth, we have changed the zero-crossing duration parameter $\tau_{p}$ in the filter's impulse response over a wide range and assessed the quality of the obtained pulses with the metric $M^{2}=\int \mathrm{d} t\left(|\psi|^{2}-|\chi|^{2}\right)^{2} / \int \mathrm{d} t|\psi|^{4}$. Here, $\psi$ is the pulse being evaluated, and $\chi$ is the theoretical sinc function (Equation (3)) with the same peak amplitude. In each case, we have verified that the spectrum was close to the ideal rectangular one. In Figure 5, we summarise the results obtained in the absence of in-cavity dispersion control. It is seen that when a flat-top spectral profile is applied on the filter (Figure 5a), the quality of the generated sinc pulses improves with increasing values of the filter bandwidth, which enable increasingly higher values of the nonlinear phase shift accumulated in the fibre. This confirms that the pulse-shaping mechanism of our laser design requires SPM and gets stronger with increasing pulse intensity and nonlinear phase shift. The peak nonlinear phase shift accumulated by the pulse, however, saturates to about $3.5 \mathrm{rad}$ in our laser configuration. Above this value, the pulse spectrum after the fibre begins to split and oscillations begin to appear in its central part. Sinc-shaped pulses with low $M$ values $(M \leq 0.06)$ are obtained over the bandwidth range from a few hundred gigahertz to a few terahertz. At smaller bandwidths, in order to enhance the spectral breathing, hence, the filtering process, more nonlinear pulse propagation is necessary in the cavity. To this end, one may either use a longer gain fibre segment or incorporate a short segment of passive nonlinear fibre into the cavity [36]. Our results [36] confirmed that high-quality sinc-shaped pulses can indeed be obtained over the bandwidth range from a few tens to a few hundreds of gigahertz in this case. For larger bandwidths than the upper bound of the interval shown in Figure 5, stable single-pulsing was not possible. On the other hand, when the enhanced spectral filtering strategy is deployed, misfit values to a sinc pulse shape below 0.03 are possible over the full bandwidth range being studied (Figure 5b). Remarkably, the misfit parameter is reduced by a factor of more than ten (and even reaching a hundred in some cases) with respect to the rectangular filtering approach for bandwidths between the lower bound of the interval studied $(10 \mathrm{GHz})$ and more than two terahertz. This relaxes the need for large nonlinear phase accumulation or, in other words, large spectral broadening in the fibre section to obtain a fairly flat pulse spectrum within the filter bandwidth. Indeed, a higher degree of concavity of the output spectrum from the fibre stemming from a lower extent of nonlinear spectral expansion can be offset by a higher value of the correction factor $\alpha$ (Figure 4). The increase of the misfit parameter with increasing bandwidth after approximately $1.5 \mathrm{THz}$ is due to the fact that at such large bandwidths, some structure of the nonlinearly-broadened pulse spectrum at the fibre output falls within the filter bandwidth. Such a structure cannot be efficiently cancelled by applying a simple convex function. Consequently, the strength of the corrective spectral shaping approach increasingly diminishes with increasing bandwidth, as is evident from the misfit parameter increasingly approaching the values obtained with the basic shaping approach. Ultimately, the corrective approach becomes dispensable. However, within such a bandwidth range, the generated sinc pulses with the basic approach are already of very high quality (Figure 5a) and, thus, do not compulsorily require corrections.

The operation of the laser under different dispersion conditions revealed a typical scenario of when a corrective Gaussian-top profile is applied on the filter: when anomalous GVD is added into the cavity by the filter, a larger shape correction factor $\alpha$ can compensate for the higher degree of concavity of the pulse spectrum at the output of the fibre to some extent, and so, better quality sinc-shaped pulses can be obtained compared to the basic filtering method. On the contrary, with increasing normal dispersion 
in the cavity, the pulse spectrum at the output of the fibre acquires an increasing convex feature, typical of DS mode-locking [12,18], which cannot be offset by a concave filter's spectral profile $(\alpha<0)$. Therefore, the improvement in pulse quality brought about by the corrective method using small negative values of $\alpha$ is minimal. We also confirmed that third-order dispersion (TOD) does not limit the operation or performance of our Nyquist laser for typical values of the TOD coefficient of the fibre.
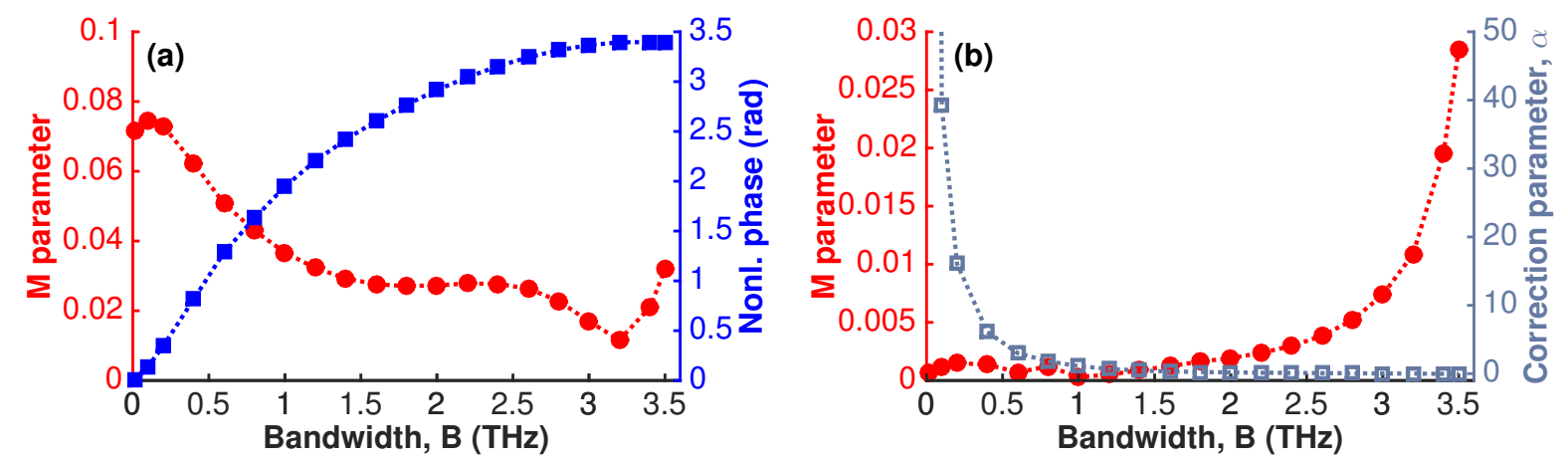

Figure 5. Misfit parameter to a sinc shape at the output of the laser (red circles) versus filter bandwidth $B$ for (a) a flat-top and (b) a Gaussian-top spectral pulse shaper and a laser without in-cavity dispersion control. The evolutions of the peak nonlinear phase shift (blue squares) and the correction factor $\alpha$ (grey open squares) are also shown. Adapted from [36]. Copyright 2015, IEEE.

\section{Dispersion-Managed Versatile Ultrafast Fibre Laser}

In this section, we review the operation of an ultrafast passively-mode-locked fibre laser, which can be readily and reliably switched among conventional soliton, stretched-pulse and DS mode-locking regimes by changing the dispersion and bandwidth of an in-cavity programmable filter only, while no changes are made to the physical layout of the laser cavity [37].

\subsection{Setup and Experimental Results}

Figure 6 shows a schematic of our passively-mode-locked EDF ring laser. The mode-locking mechanism was nonlinear polarisation rotation (NPR), facilitated through the inclusion of a combination of two polarisation controllers (PCs) and an in-fibre polarisation-dependent isolator. The EDF was $1.2 \mathrm{~m}$ long $\left(\beta_{2}=65.05 \mathrm{fs}^{2} / \mathrm{mm}\right.$ at $\left.1550 \mathrm{~nm}\right)$ and was pumped through a 980/1550 wavelength-division multiplexer by a 976-nm laser diode providing up to $300 \mathrm{~mW}$ optical power. Additional anomalous-dispersion fibre associated with the various components of the laser cavity amounted to a length of $\sim 8 \mathrm{~m}$. A programmable LCoS phase and amplitude filter was used to realise different regimes of pulse generation in the cavity. Under mode-locking conditions, the laser operated at a repetition period of $89 \mathrm{~ns}$ (Figure 6b), which remained constant throughout the experiments described below (since there was no change in the optical length of the cavity). The net dispersion of the cavity was adaptively managed by changing the curvature of the parabolic spectral phase profile of the filter (Section 2). As pulse formation at net anomalous or slightly normal GVD does not depend on 
spectral filtering, for such dispersion regimes, the amplitude filtering ability of the filter was not used: we employed the default flat-top filter's shape with the maximum achievable bandwidth. On the other hand, for the operation of the laser in the strong net-normal dispersion regime, the filter was programmed to have a narrow-bandwidth Gaussian spectral profile, as detailed below. Two 90:10 fibre couplers were employed to tap $10 \%$ of laser power out of the cavity, where one was for laser outputs after the NPR components; the other one was located after the programmable filter for investigating the pulse dynamics inside the laser cavity. The output port was connected by a 3-m long segment of standard telecom fibre $\left(\beta_{2}=-22.8 \mathrm{fs}^{2} / \mathrm{mm}\right)$ to an optical spectrum analyser, an auto-correlator and a fast photo-detector to characterise the pulses.

When there was no dispersion applied on the programmable filter, the cavity had an anomalous net dispersion. By properly adjusting the two PCs in the system, stable mode-locked pulses could be obtained (Figure 6b). The characterisations of the laser output are given in Figure 7 (blue curves) for an average pulse power of $0.15 \mathrm{~mW}$ after the fibre of the output port. The optical spectrum profile, centred at $\sim 1560 \mathrm{~nm}$ and with a spectral bandwidth at FWHM of $4.7 \mathrm{~nm}$, exhibits Kelly side-bands [48], indicating the fundamental soliton shape of the output pulse. The net dispersion of the cavity, calculated from the side-bands, is $-0.3967 \mathrm{ps}^{2}$ (at $1555 \mathrm{~nm}$ ). The measured autocorrelation trace of the pulse corresponds to an FWHM pulse duration of $0.8 \mathrm{ps}$ when a hyperbolic secant fit is assumed. This gives a time-bandwidth product (TBP) of 0.46 (at $1560 \mathrm{~nm}$ ), indicating that the pulse is slightly chirped. The soliton-like single-pulse generation regime was observed for a pump power of 20 to $26 \mathrm{~mW}$ and bore an average output power of 0.12 to $0.21 \mathrm{~mW}$, corresponding to a pulse energy of 10.7 to $18.7 \mathrm{pJ}$. Multiple solitons were generated in the system for pump powers above $26 \mathrm{~mW}$. When some normal dispersion was applied on the filter while keeping the net cavity dispersion anomalous, the spectral width of the pulses increased, which is in agreement with soliton-type pulse behaviour [49].

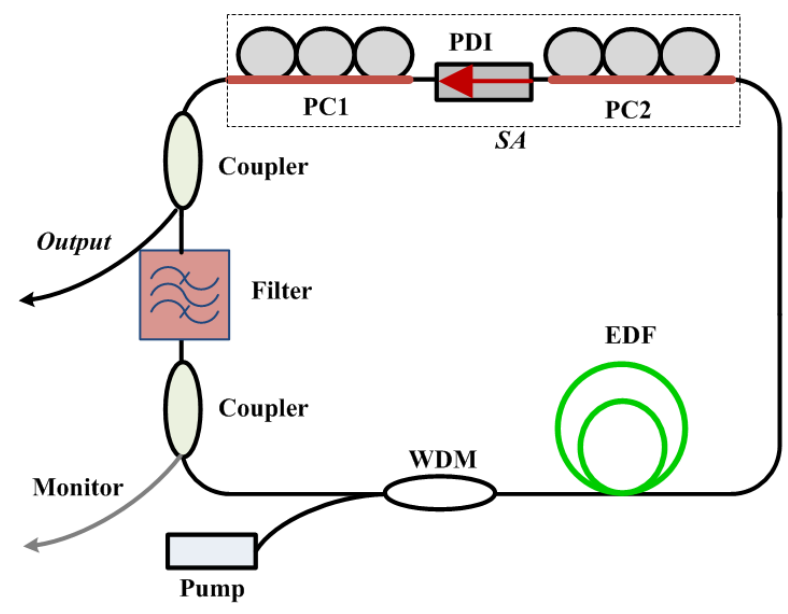

(a)

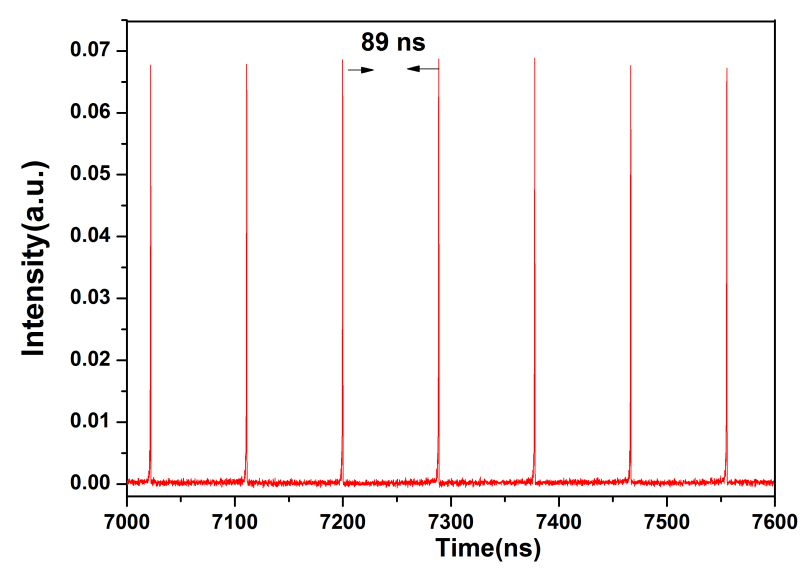

(b)

Figure 6. (a) Schematic of a filter-based dispersion-managed passively-mode-locked EDF laser. WDM: wavelength-division multiplexer; EDF: erbium-doped fibre; PC: polarisation controller; PDI: polarisation-dependent isolator; SA: saturable absorber. (b) Typical output pulse train observed on the oscilloscope. 


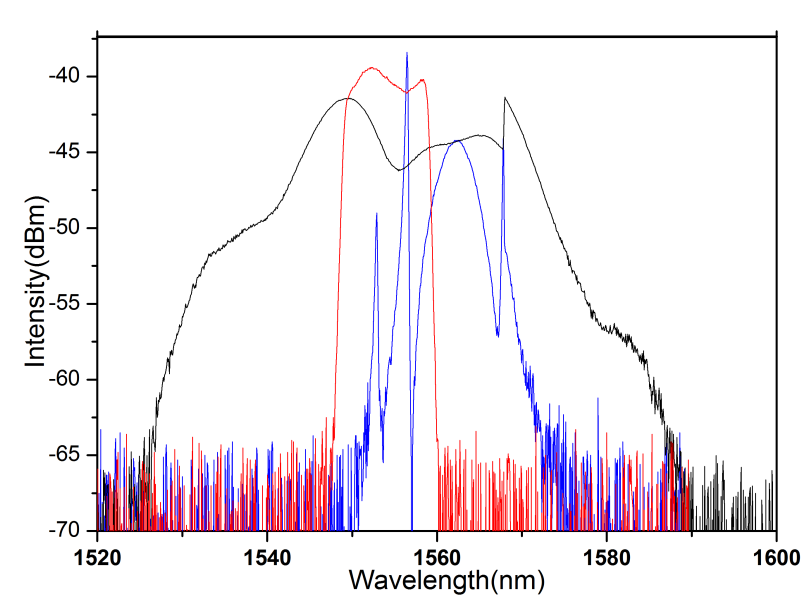

(a)

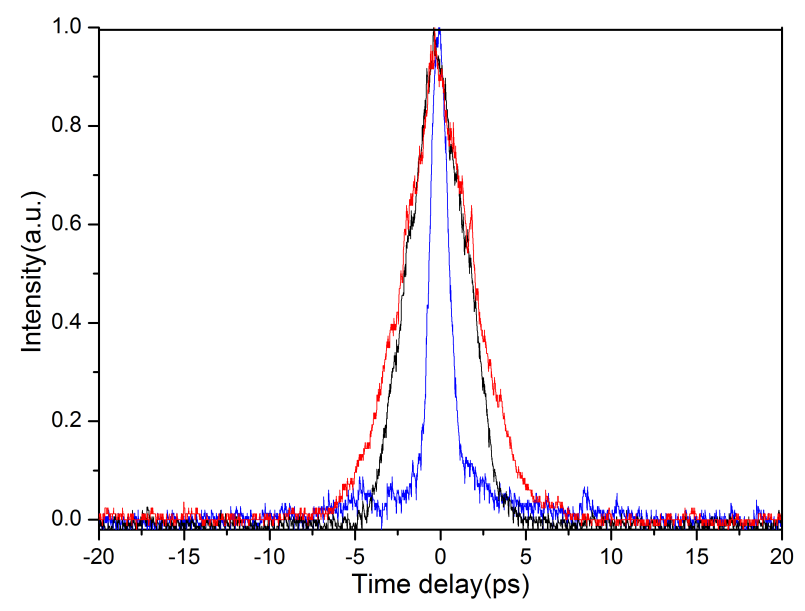

(b)

Figure 7. Typical (a) measured optical spectra and (b) autocorrelation traces for the output soliton (blue), dispersion-managed (DM) soliton (black) and dissipative soliton (DS) (red). Adapted from [37].

Next, we applied a dispersion of $0.385 \mathrm{ps}^{2}$ (at $1555 \mathrm{~nm}$ ) on the filter to shift the net cavity GVD close to a zero value. In this case, after adjustment of the PCs, the laser generated pulses (Figure 7, black curves) with a Kelly side-band-free, wide spectrum, which is a signature of the DM soliton operation regime. An even wider output pulse spectrum was obtained by establishing the net GVD to be slightly normal [49]. Single-pulse DM soliton mode locking existed under a pump power of 28.5 to $42 \mathrm{~mW}$ and bore an average output power of 0.24 to $0.41 \mathrm{~mW}$, yielding a pulse energy of 21 to $36 \mathrm{pJ}$, which is significantly higher than that of soliton-like mode locking. A further increase in the pump power resulted in multiple pulse generation. The autocorrelation trace of the pulse with $0.20 \mathrm{~mW}$ of average power after the fibre of the laser output port indicates an FWHM pulse duration of 2.78 ps when a Gaussian fit is assumed.

It is well known that DSs occur in all-normal [12,13] or strong net-normal dispersion cavities [14-16]. In our laser design, strong net-normal dispersion can be achieved by configuring the dispersion of the in-cavity filter; meanwhile, the filter bandwidth also needs to be properly controlled, as DSs require additional amplitude modulation besides saturable absorber action [12,18]. Self-starting mode-locked operation was achieved by adjustment of the PCs with a filter bandwidth of $0.8 \mathrm{THz}$. No stable mode-locked pulses were found for larger bandwidths, consistent with the discussion above. The characterisations of the laser output are given in Figure 7 (red curves) for a net GVD of $0.502 \mathrm{ps}^{2}$ in the cavity and $0.48 \mathrm{~mW}$ of average pulse power after the fibre of the output port. The output pulse spectrum is steep at the edges and has a dip in the top, typical characteristics of DS lasing [12,18]. The spectral width at FWHM is $9.7 \mathrm{~nm}$. Incidentally, we also note that the spectrum is centred at the central wavelength of the filter $(1555 \mathrm{~nm})$, which confirms the ability of an in-cavity programmable filter to tune the laser wavelength [31]. The average output power for the DS mode-locking regime was 0.45 to $0.69 \mathrm{~mW}$ under a pump power of 56.5 to $72 \mathrm{~mW}$, giving a pulse energy of 39 to $61 \mathrm{pJ}$. The pulse autocorrelation trace corresponds to an FWHM pulse duration of $3.5 \mathrm{ps}$ when a Gaussian fit is assumed. This gives a TBP of 4.21 (at $1555 \mathrm{~nm}$ ), indicating that the pulse is highly chirped inside the cavity $[12,18]$. 
Laser-generated DSs were found to exist in the net cavity dispersion range of 0.247 to $0.505 \mathrm{ps}^{2}$, and their spectral width decreased when the in-cavity GVD was increased, as was expected.

\subsection{Numerical Simulations}

To gain deeper understanding of the laser dynamics, we performed numerical simulations of the laser based on the non-distributed model described in Section 2. The laser configuration used in the simulations was the same as the experimental setup. We used a rectangular spectral profile for the filter $R(f)=\operatorname{rect}(f / B)$ to operate the laser at anomalous or slightly normal dispersion and a Gaussian spectral profile $R(f)=\exp \left[\left(-f^{2}\right) /\left(\left(2 B^{2}\right)\right)\right]$ for the strong net-normal dispersion regime, in accordance with the experiment. To account for the additional length of anomalous-dispersion fibre in the cavity, we included two segments of SMF after the SA element and the filter, respectively. Linear losses of $10 \%$ were imposed after the SMF segment following the SA and after the filter, which summarised intrinsic losses and output coupling. The laser output was monitored behind the coupler at the exit of the SMF segment following the SA, after further propagation in a $3 \mathrm{~m}$-long segment of SMF, as in the case of the experiment. The parameters used in the numerical simulations were similar to their nominal or estimated experimental values. Typical evolutions of pulse duration and spectral width in the cavity in the three pulse-generation regimes are shown in Figure 8. The respective net GVDs of the cavity were $-0.397 \mathrm{ps}^{2}, 0 \mathrm{ps}^{2}$ and $0.502 \mathrm{ps}^{2}$, which are similar values to those of the experimental results in Figure 7. The corresponding simulated pulses and spectra were in qualitative fairly good agreement with their experimental counterparts, with TBP values close to the experimental ones. Distinctly different types of evolutions can be seen from Figure 8. The soliton-like pulse is nearly static in the cavity, with temporal and spectral breathing ratios (defined as the ratios of maximum and minimum rms widths within a round-trip in the cavity) of only 1.3 and 1.2, respectively. The DM soliton experiences the largest temporal and spectral breathing [50], with the respective breathing ratios of 7.5 and 1.8. This pulse temporally stretches and compresses three times per round-trip, reaches a minimum duration in the middle of each of the EDF and the two SMF segments and acquires both signs of chirp. The mode-locked pulse at large net-normal dispersion exhibits the features of a DS with an evolution defined by the dispersion map [15]. The evolution in each fibre segment is monotonic; the pulse duration increases in the EDF segment and decreases in the SMF segments; and the spectrum, cut away by the filter, grows back in the fibre sections. The pulse is positively chirped throughout the cavity, and it depends strongly on dissipative effects, such as the spectral filter and the SA. The different roles played by spectral filtering in the DM soliton and DS mode-locking regimes are evident: in the DM soliton regime, the large filter has nearly no effect on the pulse spectrum, while the pulse is temporally compressed by the effect of the normal dispersion applied on the filter; in the DS regime, by contrast, the narrow filter cuts away the spectral structure, and consequently, the pulse duration is increased. 


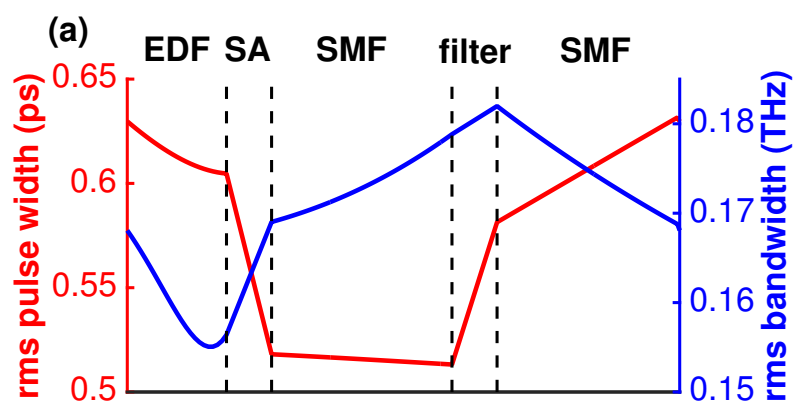

(b)

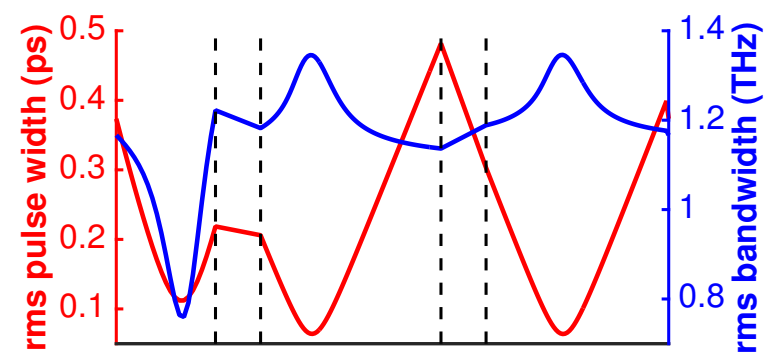

(c)

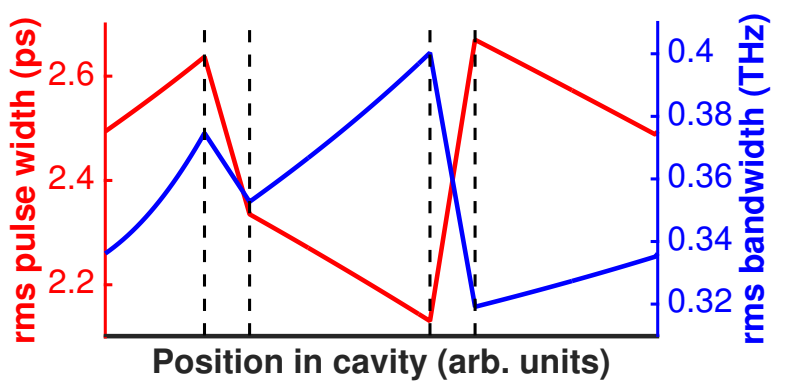

Figure 8. Simulated evolution of the rms temporal (red) and spectral (blue) widths of the pulse along the cavity for the (a) soliton, (b) DM soliton and (c) DS regimes. Adapted from [37].

\section{Conclusions}

We have provided an overview of our recent results and advances in the realisation of pulse shaping in passively-mode-locked fibre lasers by inclusion of an amplitude and phase spectral filter into the laser cavity. We have presented a fibre laser design in which pulse shaping occurs through filtering of a spectrally nonlinearly-broadened pulse in the cavity. This strategy of pulse shaping has been illustrated through the numerical demonstration of the laser operation in different pulse-generation regimes, including bright and dark parabolic, flat-top, triangular and saw-tooth waveform generations, depending on the amplitude profile of the in-cavity spectral filter. These results are of particular relevance with the high interest in the generation of specialised pulse waveforms for applications in optical signal processing and manipulation. Using a spectral filter based on a programmable optical processor, one would be able to easily reconfigure the operation of the laser with a computer interface. The pulse-shaping approach presented here is attractive because it provides a high degree of control over the output pulse shape of the laser to adjust its operation to different experimental requirements. As an 
application of this method, we have numerically shown the possibility of directly generating sinc-shaped Nyquist pulses of high quality from a laser incorporating a simple flat-top spectral filter in the cavity. We have also shown that the use of a filter's profile with a corrective convex top can compensate for the concavity of the nonlinearly-broadened pulse spectrum in the fibre, thereby relaxing the need for large nonlinear phase accumulation. The filtering process being used enables the tunability of the spectral bandwidth of the output sinc pulses over a wide range, up to a few terahertz. The high flexibility of the bandwidth can be of particular interest for photonic-assisted technologies, such as photonic analogue-to-digital conversion or optical sampling. Finally, we have reported on a recently-developed versatile ultrafast passively-mode-locked EDF laser in which the pulse formation mechanism can be flexibly controlled by an in-cavity programmable filter. We have demonstrated that conventional solitons, DM solitons and DSs can be selected and reliably targeted in a single laser by adaptively changing the dispersion and bandwidth programmed on the in-cavity filter. We have confirmed the different regimes of pulse generation experimentally and by numerical simulations. The transitions among the different pulse-shaping regimes are easily reconfigurable and inherently interesting in the field of ultrafast optics owing to their vastly different characteristics. This multifunction ultrashort-pulse fibre laser source has great potential for several applications, such as optical communications and signal processing, and can also be readily implemented at different wavelength regions, such as at the two-micron region, where suitable dispersion-compensating fibres are still under development. From a fundamental standpoint, our work confirms the great potential of the concept of an in-cavity spectral pulse shaper for manipulating and controlling the dynamics of mode-locked fibre lasers and, thus, enabling different mode-locking regimes.

\section{Acknowledgments}

We acknowledge important contributions of our colleagues, Sergei K. Turitsyn and Periklis Petropoulos, to the original papers discussed in this review. We would also like to acknowledge the financial support of the Leverhulme Trust (Grant RPG-278) and the European Commission Marie Curie International Incoming Fellowship scheme (Grant No. 628198).

\section{Author Contributions}

Sonia Boscolo had the initial idea for and led the work presented in this paper. She contributed to the design of the study, the production of the numerical results, the analysis and interpretation of the results and the preparation of the manuscript. Junsong Peng contributed to the production, analysis and interpretation of the experimental results and the preparation of the manuscript. Christophe Finot contributed to the design of the study, the analysis and interpretation of the results and the preparation of the manuscript.

\section{Conflicts of Interest}

The authors declare no conflict of interest. 


\section{References}

1. Keller, U. Recent developments in compact ultrafast lasers. Nature 2003, 424, 831-838.

2. Sibbett, W.; Lagatsky, A.; Brown, C. The development and application of femtosecond laser systems. Opt. Express 2012, 20, 6989-7001.

3. Renninger, W.H.; Chong, A.; Wise, F.W. Pulse shaping and evolution in normal-dispersion mode-locked fiber lasers. IEEE J. Sel. Top. Quantum Electron. 2012, 18, 389-398.

4. Duling, I.N. All-fiber ring soliton laser mode locked with a nonlinear mirror. Opt. Lett. 1991, 16, 539-541.

5. Tamura, K.; Ippen, E.P.; Haus, H.A.; Nelson, L.E. 77-fs pulse generation from a stretched-pulse mode-locked all-fiber ring laser. Opt. Lett. 1993, 18, 1080-1082.

6. Turitsyn, S.K.; Bale, B.G.; Fedoruk, M.P. Dispersion-managed solitons in fibre systems and lasers. Phys. Rep. 2012, 521, 135-203.

7. Ilday, F.Ö.; Buckley, J.R.; Clark, W.G.; Wise, F.W. Self-similar evolution of parabolic pulses in a laser. Phys. Rev. Lett. 2004, 92, doi:10.1103/PhysRevLett.92.213902.

8. Oktem, B.; Ülgüdür, C.; Ilday, F.Ö. Soliton-similariton fibre laser. Nat. Photonics 2010, 4, $307-311$.

9. Zhang, Z.; Öktem, B.; Ilday, F.Ö. All-fiber-integrated soliton-similariton laser with in-line fiber filter. Opt. Lett. 2012, 37, 3489-3491.

10. Renninger, W.H.; Chong, A.; Wise, F.W. Self-similar pulse evolution in an all-normal-dispersion laser. Phys. Rev. A 2010, 82, doi:10.1103/PhysRevA.82.021805.

11. Aguergaray, C.; Méchin, D.; Kruglov, V.; Harvey, J.D. Experimental realization of a mode-locked parabolic Raman fiber oscillator. Opt. Express 2010, 18, 8680-8687.

12. Chong, A.; Buckley, J.; Renninger, W.; Wise, F. All-normal-dispersion femtosecond fiber laser. Opt. Express 2006, 14, 10095-10100.

13. Renninger, W.H.; Chong, A.; Wise, F.W. Giant-chirp oscillators for short-pulse fiber amplifiers. Opt. Lett. 2008, 33, 3025-3027.

14. Cabasse, A.; Ortaç, B; Martel, G.; Hideur, A.; Limpert, J. Dissipative solitons in a passively mode-locked Er-doped fiber with strong normal dispersion. Opt. Express 2008, 16, 19322-19329.

15. Bale, B.G.; Boscolo, S.; Turitsyn, S.K. Dissipative dispersion-managed solitons in mode-locked lasers. Opt. Lett. 2009, 34, 3286-3288.

16. Peng, J.; Zhan, L.; Gu, Z.; Qian, K.; Hu, X.; Luo, S.; Shen, Q. Direct generation of 4.6-nJ 78.9-fs dissipative solitons in an all-fiber net-normal-dispersion Er-doped laser. IEEE Photon. Technol. Lett. 2012, 24, 98-100.

17. Li, H.; Liu, J.; Cheng, Z.; Xu, J.; Tan, F.; Wang, P. Pulse-shaping mechanisms in passively mode-locked thulium-doped fiber lasers. Opt. Express 2015, 23, 6292-6303.

18. Wise, F.W.; Chong, A.; Renninger, W.H. High-energy femtosecond fiber lasers based on pulse propagation at normal dispersion. Laser Photon. Rev. 2008, 2, 58-73. 
19. Boscolo, S.; Fatome, J.; Turitsyn, S.K.; Millot, G.; Finot, C. Temporal and spectral nonlinear pulse shaping methods in optical fibres. In All-Optical Signal Processing: Data Communication and Storage Applications; Wabnitz S., Eggleton, B.J., Eds.; Springer: Cham, Switzerland, 2015; pp. $105-128$.

20. Ortaç, B.; Plötner, M.; Schreiber, T.; Limpert, J.; Tünnermann, A. Experimental and numerical study of pulse dynamics in positive net-cavity dispersion mode-locked $\mathrm{Yb}$-doped fiber lasers. Opt. Express 2007, 15, 15595-15602.

21. Chernysheva, M.A.; Krylov, A.A.; Ogleznev, A.A.; Arutyunyan, N.R.; Pozharov, A.S.; Obraztsova, E.D.; Dianov, E.M. Transform-limited pulse generation in normal cavity dispersion erbium doped single-walled carbon nanotubes mode-locked fiber ring laser. Opt. Express 2012, 20, 23994-24001.

22. Nishizawa, N.; Jin, L.; Kataura, H.; Sakakibara, Y. Dynamics of a dispersion-managed passively mode-locked Er-doped fiber laser using single wall carbon nanotubes. Photonics 2015, 2, 808-824.

23. Gumenyuk, R.; Vartiainen, I.; Tuovinen, H.; Kivistö, S.; Chamorovskiy, Y.; Okhotnikov, O.G. Dispersion compensation technologies for femtosecond fiber system. Appl. Opt. 2011, 50, 797-801.

24. Boscolo, S.; Turitsyn, S.K. Intermediate asymptotics in nonlinear optical systems. Phys. Rev. A 2012, 85, doi:10.1103/PhysRevA.85.043811.

25. Peng, J.; Zhan, L.; Gu, Z.; Qian, K.; Luo, S.; Shen, Q. Experimental observation of transitions of different pulse solutions of the Ginzburg-Landau equation in a mode-locked fiber laser. Phys. Rev. A 2012, 86, doi:10.1103/PhysRevA.86.033808.

26. Weiner, A.M. Femtosecond pulse shaping using spatial light modulators. Rev. Sci. Instrum. 2000, 71, 1929-1960.

27. Brixner, T.; Damrauer, N.H.; Niklaus, P.; Gerber, G. Photoselective adaptive femtosecond quantum control in the liquid phase. Nature 2001, 414, 57-60.

28. Dudovich, N; Oron, O.; Silberberg, Y. Single-pulse coherently controlled nonlinear Raman spectroscopy and microscopy. Nature 2002, 418, 512-514.

29. Roelens, M.A.F.; Frisken, S.; Bolger, J.A.; Abakoumov, D.; Baxter, G.; Poole, S.; Eggleton, B.J. Dispersion trimming in a reconfigurable wavelength selective switch. J. Lightwave Technol. 2008, $26,73-78$.

30. Hillerkuss, D.; Schmogrow, R.; Schellinger, T.; Jordan, M.; Winter, M.; Huber, G.; Vallaitis, T.; Bonk, R.; Kleinow, P.; Frey,F.; et al. 26 Tbit s-1 line-rate super-channel transmission utilizing all-optical fast Fourier transform processing. Nat. Photonics 2011, 5, 364-371.

31. Schröder, J.; Vo, T.D.; Eggleton, B.J. Repetition-rate-selective, wavelength-tunable mode-locked laser at up to $640 \mathrm{GHz}$. Opt. Lett. 2009, 34, 3902-3904.

32. Yang, X.; Hammani, K.; Richardson, D.J.; Petropoulos, P. Passively mode-locked fiber laser incorporating adaptive filtering and dispersion management. In Proceedings of the Conference on Lasers and Electro-Optics, OSA Technical Digest, Optical Society of America, San Jose, CA, USA, 9-14 June 2013; pp. 1-2.

33. Schröder, J.; Coen, S.; Sylvestre, T.; Eggleton, B.J. Dark and bright pulse passive mode-locked laser with in-cavity pulse shaper. Opt. Express 2010, 18, 22715-22721. 
34. Boscolo, S.; Finot, C.; Karakuzu, H.; Petropoulos, P. Pulse shaping in mode-locked fiber laser by in-cavity spectral filter. Opt. Lett. 2014, 39, 438-441.

35. Boscolo, S.; Finot, C. Nonlinear pulse shaping in fibres for pulse generation and optical processing. Int. J. Opt. 2012, 2012, doi: 10.1155/2012/159057.

36. Boscolo, S.; Finot, C.; Turitsyn, S.K. Bandwidth programmable optical Nyquist pulse generation in passively mode-locked fiber laser. IEEE Photon. J. 2015, 7, doi:10.1109/JPHOT.2015.2475177.

37. Peng, J.; Boscolo, S. Switching among pulse-generation regimes in passively mode-locked fibre laser by adaptive filtering. SPIE Photonics Europe, Submitted.

38. Haus, H.A. Mode-locking of lasers. IEEE J. Sel. Top. Quantum Electron. 2000, 6, 1173-1185.

39. Finot, C.; Millot, G. Synthesis of optical pulses by use of similaritons. Opt. Express 2004, 12, 5104-5109.

40. Leuthold, J.; Freude, W. Optical OFDM and Nyquist multiplexing. In Optical Fiber Telecommunications V1B; Kaminov, I.P., Lee, T., Willner, A.E., Eds.; Elsevier: Oxford, UK, 2013; pp. 381-432.

41. Valley, G.C. Photonic analog-to-digital converters. Opt. Express 2007, 15, 1955-1982.

42. Supradeepa, V.R.; Long, C.M.; Wu, R.; Ferdous, F.; Hamidi, E.; Leaird, D.E.; Weiner, A.M. Comb-based radiofrequency photonic filters with rapid tunability and high selectivity. Nat. Photon. 2012, 6, 186-194.

43. Preußler, S.; Jamshidi, K.; Wiatrek, A.; Henker, R.; Bunge, C.-A.; Schneider, T. Quasi-light-storage based on time-frequency coherence. Opt. Express 2009, 17, 15790-15798.

44. Nakazawa, M.; Hirooka, T.; Ruan, P.; Guan, P. Ultrahigh-speed “orthogonal” TDM transmission with an optical Nyquist pulse train. Opt. Express 2012, 20, 1129-1140.

45. Vedadi, A.; Shoaie, M.A.; Brès, C.-S. Near-Nyquist optical pulse generation with fiber optical parametric amplification. Opt. Express 2012, 20, B558-B565.

46. Soto, M.A.; Alem, M.; Shoaie, M.A.; Vedadi, A.; Brès, C.-S.; Thévenaz, L.; Schneider, T. Optical sinc-shaped Nyquist pulses of exceptional quality. Nat. Commun. 2013, 4, 3898.

47. Nakazawa, M.; Yoshida, M.; Hirooka, T. The Nyquist laser. Optica 2014, 1, 15-22.

48. Kelly, S. Characteristic side-band instability of periodically amplified average soliton. Electron. Lett. 1992, 28, 806-807.

49. Nelson, L.E.; Jones, D.J.; Tamura, K.; Haus, H.A.; Ippen, E.P. Ultrashort-pulse fiber ring lasers. Appl. Phys. B-Lasers Opt. 1997, 65, 277-294.

50. Chouli, S.; Soto-Crespo, J.M.; Grelu, P. Optical spectra beyond the amplifier bandwidth limitation in dispersion-managed mode-locked fiber lasers. Opt. Express 2011, 19, 2959-2964.

(C) 2015 by the authors; licensee MDPI, Basel, Switzerland. This article is an open access article distributed under the terms and conditions of the Creative Commons Attribution license (http://creativecommons.org/licenses/by/4.0/). 